Randomized Trial

\title{
The Effects of Radiofrequency Neurotomy Using a Strip-Lesioning Device on Patients with Sacroiliac Joint Pain: Results from a Single-Center, Randomized, Sham-Controlled Trial
}

Vivek Mehta, MD, FRCA ${ }^{1}$, Kavita Poply, FRCA ${ }^{1}$, Michael Husband, FRCA ${ }^{2}$,

Sibtain Anwar, PhD, FRCA ${ }^{3}$, and Richard Langford, FRCA ${ }^{1}$

From: ${ }^{1}$ Pain and Anaesthesia Research Centre, St.

Bartholomew's Hospital, United Kingdom; ${ }^{2}$ St. Richard's Hospital, Western Sussex Hospital NHS Trust, Spitafield Lane, Chicester, West Sussex; ${ }^{3}$ Department of Perioperative Medicine, Barts Heart Centre, Barts Health NHS Trust, West Smithfield London

Address Correspondence: Vivek Mehta, MD, FRCA Pain and Anaesthesia Research Centre

St. Bartholomew's Hospital West Smithfield London, $\mathrm{EC}_{1} \mathrm{~A} 7 \mathrm{BE}$ United Kingdom E-mail:

vivek.mehta@mac.com

Disclaimer: There was no external funding in the preparation of this manuscript.

Conflict of interest: Each author certifies that he or she, or a member of his or her immediate family, has no commercial association (i.e., consultancies, stock ownership, equity interest, patent/licensing arrangements, etc.) that might pose a conflict of interest

in connection with the submitted manuscript.

Manuscript received: o9-10-2017

Revised manuscript received: 03-27-2018

Accepted for publication: 03-29-2018

Free full manuscript: www.painphysicianjournal.
Background: Radiofrequency neurotomy (RFN) is a therapy aimed at providing lasting back pain relief for sacroiliac joint (SIJ) pain. A recent advancement in RFN is a strip lesioning technique that involves placement of a single curved electrode and a 3-pole design that facilitates the creation of 5 overlapping lesions. These lesions form one long strip lesion accessible through a single entry point, without the need for multiple punctures. Although the early case series data looks promising, there is lack of long-term, randomized, controlled study evaluating the strip-lesioning system for SIJ pain.

Objectives: The purpose of this study was to examine the safety and effectiveness of RFN using a strip lesioning device for reduction of SIJ pain.

Study Design: Prospective, double-blind, randomized, sham-controlled trial with 6-month follow-up

Setting: A tertiary care interventional pain management center in the UK

Methods: Patients with SIJ pain with positive diagnostic local anesthetic blocks were randomly assigned (2:1) to either the sham (no RF lesions performed) or the active group (RF lesions performed).

The primary endpoint was improvement of pain using the Numeric Rating Scale (NRS-11) at 3 months. Results were analyzed using nonparametric tests. Safety, secondary, and long-term outcome data were also collected.

Results: Seventeen of 30 enrolled patients were randomly assigned to active treatment ( $n=$ 11) or sham treatment $(n=6)$. At 3 months, the mean NRS-11 score for the active group had decreased significantly, from $8.1( \pm 0.8)$ at baseline to $3.4( \pm 2.0)(P<0.001)$. The sham group did not experience a statistically or clinically meaningful decrease in mean NRS-11 score from baseline $(7.3 \pm 0.8)$ to 3 months $(7.0 \pm 1.7)$. On average, patients in the active group moved from borderline anxiety at baseline $(9.4 \pm 5.9)$ to no anxiety $(6.6 \pm 6.3)$ at 3 months. Results were similar at 6 months.

Limitations: Recruitment was stopped at 30 enrolled patients, only 17 of whom were randomly assigned to active or sham treatment, after the interim analysis indicated a statistically significant ( $P$ $<0.001)$ difference in the pain outcome between the treatment and the sham groups.

Conclusions: This study demonstrated that radiofrequency neurotomy using a strip lesioning device is an appropriate therapy to treat SIJ pain.

Key words: Radiofrequency, sacroiliac joint pain, low back pain, neurotomy, randomized controlled trial, simplicity

ClinicalTrials.gov registration: NCT01726608; NRES REC ref 12/LO/1312

Pain Physician 2018: 21:607-618 
T he sacroiliac joint (SIJ) is the largest axial joint in the body and is susceptible to trauma and degeneration that can eventually lead to chronic, nonradicular pain. It has been suggested that at least $20 \%$ of low back pain can be attributed to SIJ dysfunction (1). Studies have estimated a high prevalence of SIJ pain (SIJP), producing enormous health and financial burdens on patients, their families, and society (2). Diagnosis of SIJP can itself be challenging due to lack of accurate clinical diagnostic tests or radiological features. Intraarticular comparative local anesthetic blocks are frequently used, but with only moderate validity (3). Furthermore, in a recent systematic review, a double-block paradigm (using $2 \%$ lignocaine and $0.5 \%$ bupivacaine sequentially) has been suggested to improve identification of SIJ pain (4). Following diagnosis, conventional management of SIJP can vary from intraarticular steroid injections to physical and pharmacological treatments, showing inconsistent benefits (5). Long-term evaluations of these conventional therapies have failed to show consistent and satisfactory benefits $(6,7)$.

Radiofrequency neurotomy (RFN) is an established therapy in the clinical management of chronic nonradicular pain originating from the zygoapophyseal joints (also known as facet joints) and refractory to conventional treatment (8-11). Traditional RFN involves applying a local electrical current to the nerves that produce thermal lesions in order to disrupt the pain signals. The thermal lesions result in coagulation of both motor and sensory fibers in a nonselective manner, denaturing the nerve and interrupting transmission of the nociceptive pain signal. Traditional RFN for SIJP involves multiple injections and individual lesioning to the lateral branches of the L5 and S1, 2, and 3 nerve roots (12-14). However, this varied anatomical target field prohibits consistent device placement and focused neuronal targeting, leading to the variable efficacy reported in the literature (15-17). Alternatively, cooled RFN can produce larger thermal lesions, compensating for the variability in location of the anatomical target field. In a randomized controlled study evaluating 28 patients with SIJP, Cohen and colleagues found that patients experienced pain relief of $50 \%$ or greater with significant functional improvement at 1 , 3 , and 6 months post-procedure (18). Moreover, when compared to placebo, the cooled RFN group reported significantly greater pain relief at the primary endpoint of one month post-procedure. The authors concluded that cooled RFN provides satisfactory pain relief and functional benefits to patients suffering from SIJP. In a more recent randomized, controlled, crossover study evaluating the long-term outcomes of patients suffering from SIJP, Patel and colleagues reported significant improvement in pain, disability, function, and quality of life in 51 patients (2:1 cooled RFN/sham) at 1, 3, 6, and 9 months post-procedure. The authors also demonstrated evidence of cooled RFN efficacy at 6 and 9 months for patients in the sham group who crossed over to the treatment group at the 3-month follow-up visit (18). Patel and colleagues followed these patients up post-procedure to demonstrate efficacy persisting to the one-year follow-up visit. This suggests that cooled RFN treatment for SIJP may be an effective treatment with long-lasting therapeutic benefits (19).

Although the benefits of cooled RFN have been established in terms of decreased procedure time and improved patient outcomes, the multiple injections and entry points required for individual lesioning may be inconsistent and time consuming. A recent advancement in RFN is a strip lesioning technique, which uses a curved electrode and a 3-pole design that facilitates the creation of 5 overlapping lesions. These overlapping lesions form a long strip lesion accessible through a single entry point, without the need for multiple punctures. To date, there are very few descriptions of this new technique in the literature. A 12-month retrospective follow-up study reported a statistically significant improvement in pain scores (8.8 vs 4.3 ) and a general improvement in global health (20). Recently, a sham-controlled study from the Netherlands failed to demonstrate any benefit of the strip lesion technique. However, that study had a mixed population (including patients with radicular pain) with an unusually frequent response to diagnostic SI joint blocks ( $86.1 \%$ of the tested patients) (21). Although the descriptions are promising, there is a consistent lack of long-term randomized controlled studies evaluating the striplesioning system.

To our knowledge, our study is the first to evaluate the clinical efficacy of the strip-lesioning technique versus a sham procedure among patients suffering from SIJP in a 6-month prospective, randomized, doubleblind controlled design with strict inclusion criteria.

\section{Methods}

This study was a single-center, prospective, doubleblind, randomized (2:1), sham-controlled trial evaluating the clinical efficacy of RFN therapy in patients with SIJ pain using the Simplicity III device. Approval to con- 
duct the study was obtained from the National Research Ethics Approval system (NRES REC ref 12/LO/1312).

\section{Patients}

Table 1 summarizes the inclusion/exclusion criteria. Patients, aged 18 to 80 years, with localized unilateral lumbosacral pain, were identified and screened from the chronic pain clinics at Barts Health NHS Trust, London, UK between October 2012 and May 2015. Having been made aware of the study procedures, risks, and benefits, patients gave informed consent.

Consented patients were enrolled if they had unilateral localized lumbosacral pain of at least 3-month duration with a pain intensity score of 5 or more on the Numeric Rating Scale (NRS-11) and a positive FABER test (pain provocation on flexion, abduction, and external rotation of the hip) on clinical examination. Following enrollment, to confirm the diagnosis of SIJ pain, all patients underwent (on 2 separate occasions 4 weeks apart), 2 sets of fluoroscopic, contrast-guided diagnostic intraarticular SIJ injections (explained in surgical procedures). Patients were determined to have SIJ pain if they reported, on both occasions, at least $80 \%$ pain relief on the NRS-11 immediately following the diagnostic block; these patients continued in the study.

\section{Study Design}

Patients with positive responses to the diagnostic SIJ intraarticular injections were randomly assigned to either active (lesions performed) or sham treatment (no lesions performed) in a 2:1 randomization scheme. Both the patients and the clinical operator (VM) were blinded to the procedure. After blindly evaluating each treated patient at 3 months, they and the investigators were unblinded, allowing those in the sham group with pain (NRS-11 score of 5 or more) to be offered the active strip lesioning treatment.

All patients had additional unblinded evaluations at 6 months post-randomization.

\section{Outcomes}

Outcomes collected at all visits were:

- Numeric Rating Scale (NRS-11), an 11-point scale ranging from 0 to 10 where higher scores represent greater pain intensity

- $\quad$ 12-item Short Form Health Survey (SF12), a validated 12-item assessment of health-related quality of life with subscales consisting of a Physical Component Score (PCS) and Mental Component Score (MCS). Higher scores in these components represent better physical and mental health

- Hospital and Depression Scale (HADS), an assessment of co-existing anxiety and depression

- EuroQol (EQ-5D), which measures health-related quality of life

- $\quad$ Adverse events.

\section{Interventions}

Double intraarticular diagnostic block: Each intraarticular diagnostic block was performed in the Day Surgery Unit at St. Bartholomew's Hospital in London by a single experienced operator (VM). Patients received $2 \mathrm{~mL}$ injections of $1 \%$ lignocaine into the SIJ following confirmation of correct needle placement with radiopaque contrast-enhanced (up to $1 \mathrm{~mL}$ of $61.2 \% \mathrm{w} / \mathrm{v}$ lopamidol equivalent to $300 \mathrm{mg}$ iodine $/ \mathrm{mL}$ ) arthrogram (Fig. 1).

Table 1. Inclusion/exclusion criteria.

\section{Inclusion Criteri}

1) Written, informed consent

2) Age: $18-80$ years old

3) Low back pain of more than 6 months duration with a minimum pain intensity of greater than 5 out of 10 on an 11-point numerical rating scale in the 7 days preceding study entry.

4) Subjects must achieve greater than $80 \%$ reduction in pain following each diagnostic, intra-articular block. Subjects must undergo 2 blocks in total prior to randomisation.

5) Female subjects of potential childbearing age must be using adequate contraception (i.e. using oral or intramuscular contraception or an IUCD) and must have a negative urine test. 6) No vulnerable patient groups shall be recruited into this study

Exclusion Criteria

1) Subjects who do not fulfill inclusion criteria

2) Subjects who have previously been treated by any sacroiliac joint radiofrequency neurotomy

3) Subjects who are breastfeeding

4) Contraindications to local anaesthetics and radiofrequency

neurotomy as listed in their respective summary of product

characteristics

5) Subjects with documented or suspected alcohol or drug abuse, or who are suspected of having an addictive personality

6) Subjects to whom any of the following apply: Major trauma to the lumbar spine in the six months preceding study entry. Infection in the lumbar

spine in the six months preceding study entry

7) Subjects' known to have a condition that, in the investigator's judgment precludes entry into the study.

8) Subjects with a significant psychiatric disorder (including depression) or subjects receiving anti-psychotic medication.

9) Subjects who have received an investigational drug or have used an investigational device in the 30 days preceding study entry.

10) Subjects unable to comply with the study assessments or unable to complete the questionnaires. 
Active RFN: Active RFN included both radiofrequency lesioning of the $L 5$ medial branch of the primary dorsal root nerve and strip lesioning of the lateral branches of the S1, 2, and 3 nerve roots under local anesthesia. Radiofrequency lesioning of the L5 medial branch of the primary dorsal root nerve was performed using a $22 \mathrm{G}, 10 \mathrm{~cm}$ electrode (Neurotherm, St. Jude Medical, Abbott, St. Paul, MN) with a $10 \mathrm{~mm}$ active tip using the Spine Intervention Society technique (22). Both sensory $(<0.5 \mathrm{~V})$ and motor $(>1.5 \mathrm{~V})$ threshold testing were performed to confirm accurate placement. The strip lesion electrode (Simplicity III, St. Jude Medical, Abbott, St. Paul, MN) was then inserted, under local anesthesia, and advanced to strip lesion the lateral branches of the 1,2 , and 3 sacral nerve roots after confirmation with a negative motor stimulus (>3.0 V). All procedures were performed by an experienced single operator (VM) to avoid any inter-operator error (Fig. 2).
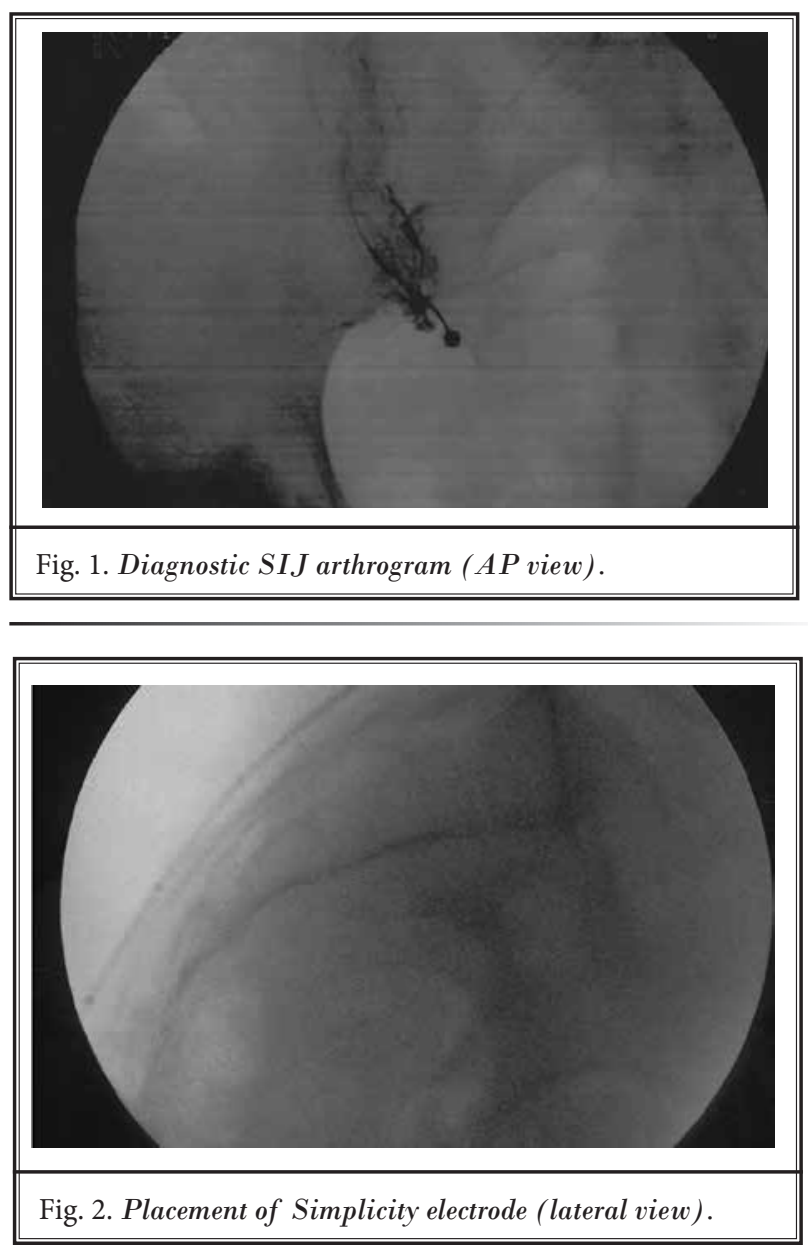

Sham Procedure: The sham procedure was identical to the active RFN treatment except that no radiofrequency energy was applied. Under local anesthesia, the L5 primary dorsal root was stimulated (both sensory $<0.5 \mathrm{~V}$, and motor $>1.5 \mathrm{~V}$, threshold testing) to confirm accurate placement and the sham procedure was performed by inserting and advancing the Simplicity III electrode. Negative motor stimulus (>3.0 V) was obtained, and the probe was placed for 5 minutes (to match the active treatment duration) without any radiofrequency energy electric current being delivered. Therefore, no lesion was produced in this group.

\section{Randomization}

The sequence of randomization was generated using an envelope system. Patients underwent their intervention as soon as possible after randomization.

\section{Sequence Generation}

The allocation ratio was 2:1 with no stratification.

\section{Allocation Concealment}

An investigator, not performing the trial intervention, randomly selected an envelope containing instructions for either a sham or active RFN procedure. These remained sealed and were handed to a single nurse operator of the console to deliver the randomized intervention.

\section{Blinding}

Following placement of the Simplicity III device, the study investigators (including VM, the operator performing the treatment) vacated the operating suite for the duration of treatment (active or sham) and therefore remained blinded to treatment allocation. In addition, the sound system of the console (which provides a bleeping sound with the delivery of radiofrequency energy) was turned off to maintain blinding of the patient and investigators.

\section{Sample Size}

There is agreement in the literature that the minimal, clinically relevant difference for pain relief on the NRS-11 is 2 points (23). Using a previous, sham-controlled study evaluating conventional RFN for SIJ disease, we calculated that a minimum of 15 treated patients in each group would allow a detection of a 2-point, minimally important clinical difference, as recorded using the NRS11 , at $80 \%$ power. An interim analysis was planned at 30 enrolled (but not necessarily randomized) patients. 


\section{Statistical Analysis}

Continuous outcomes are presented as means ( \pm SD) and categorical outcomes are presented as frequencies (proportions). The primary endpoint was the mean magnitude of change on the NRS-11 for both groups at 3 months following the intervention. This was analyzed using a repeated measures one-way ANOVA model (RMANOVA), followed by Tukey's post-hoc test for pairwise comparisons. Secondary outcomes of pain (average NRS-11 over the last 7 days) at 6 months, SF-12, HADS, and EQ-5D, were also analyzed using RMANOVA. Comparisons between the 2 groups were conducted using independent $t$ tests at 3 months. Given the relatively small sample size, a Mann-Whitney test was also conducted at 3 months to inspect differences between the groups. Adverse events (AE) were collected throughout the study and are presented as prevalence (number of patients with an AE/total number of patients). All analyses were performed using SAS Version 9.3 (SAS Institute, Inc., Cary, NC) with a type 1 error of 0.05 and adjusting for multiple comparisons.

\section{RESULTS}

\section{Patient Disposition and Demographics}

Patient progression is summarized in Fig. 3. Thirty patients with a mean NRS-11 score of $7.9 \pm 0.8$ were enrolled in the study and received their first diagnostic block. A month after the first diagnostic block, 22 patients reported a mean NRS-11 score of $7.8 \pm 0.9$ and underwent $a$ second intraarticular diagnostic block. After an additional month, 17 patients (with mean NRS-11 score of $7.8 \pm 0.8$ ) proceeded to randomization (Fig. 4). Of the 13 patients who failed to randomize, 7 failed to achieve adequate pain relief during diagnostic blocks, 3 voluntarily withdrew from the study (one after the first diagnostic, and 2 after the second diagnostic block), and 3 were lost to follow-up prior to randomization. As a result, a total of 17 patients proceeded to the randomization phase of the study, resulting in 11 active and 6 sham. The average age of the randomized patients was 58.5 years ( 56.6 years in the active group and 62.6 years in the sham group). At the 3-month follow up, when unblinding took place, 4 of the 6 patients in the sham group elected to have (nonblinded) active treatment, giving a total of 15 patients who were treated with strip lesioning for SIJP.

\section{Primary Endpoint}

At 3 months post-RFN, patients in the active group reported a mean NRS-11 score of $3.4 \pm 2.0$, compared to a pre-randomization mean NRS-11 score of $8.1 \pm 0.8$ (mean NRS reduction $=4.7 ; P<0.001$ ). Patients in the sham group, at 3 months, reported a mean NRS-11 score of $6.5 \pm 2.0$, compared to a pre-randomization mean NRS-11 score of $7.3 \pm 0.8$ (mean NRS reduction $=0.8 ; P$ $>0.01$ ). The mean difference between the treatment and sham groups at 3 months was also determined to be statistically significant $(P<0.001)$ (Fig. 5). Wilcoxon Mann-Whitney test between the 2 groups also yielded significant results at 3 months $(P=0.015)$.

\section{Secondary Endpoints: 3-Month Crossover Outcomes}

For the HAD-Anxiety scale, patients reported mean baseline scores of $9.4 \pm 5.9$ and $9.5 \pm 6.5$ for the active and sham groups, respectively (Fig. 6). At 3 months, patients in the active group reported an improved, albeit statistically insignificant, mean HAD-Anxiety score of $6.6 \pm 6.3$. In contrast, patients in the sham group reported a worse mean HAD-Anxiety score of 10.0 \pm 7.1 . For the HAD-Depression scale, patients reported mean baseline scores of $10.1 \pm 4.8$ and $9.8 \pm 5.3$ for the active and sham groups, respectively. At 3 months, patients in the active group reported an improved, albeit statistically insignificant, mean HAD-Depression score of $9.0 \pm 5.9$. Patients in the sham group did not show any improvement in their HAD-depression scores, reporting a mean score of $9.8 \pm 3.8$ at 3 months. No statistical differences were found between the active and sham groups at 3 months for either the HAD-Anxiety or the HAD-Depression scale $(P=0.1501)$.

The average EQ-5D scores at baseline were $0.16 \pm$ 0.32 and $0.45 \pm 0.15$ for the active and sham groups, respectively. At 3 months, the EQ-5D score for the active group improved to $0.48 \pm 0.29$. On the other hand, the sham group reported a lower mean EQ-5D score at 3 months: $0.38 \pm 0.21$. At baseline, patients reported a mean PCS score of $28.4 \pm 7.1$ and $28.6 \pm 5.0$ in the active and sham groups, respectively. At 3 months post-randomization, patients' mean PCS scores improved to 34.7 \pm 10.8 in the active group and $29.6 \pm 5.6$ in the sham group. For the MCS, patients reported a mean baseline score of $42.4 \pm 14.5$ and $43.7 \pm 12.4$ in the active and sham groups, respectively. At 3 months post-randomization, patients' mean MCS scores improved to $49.5 \pm$ 11.9 in the active group, and declined to $40.1 \pm 12.7$ in the sham group. Although these slight improvements were found in the physical and mental component 


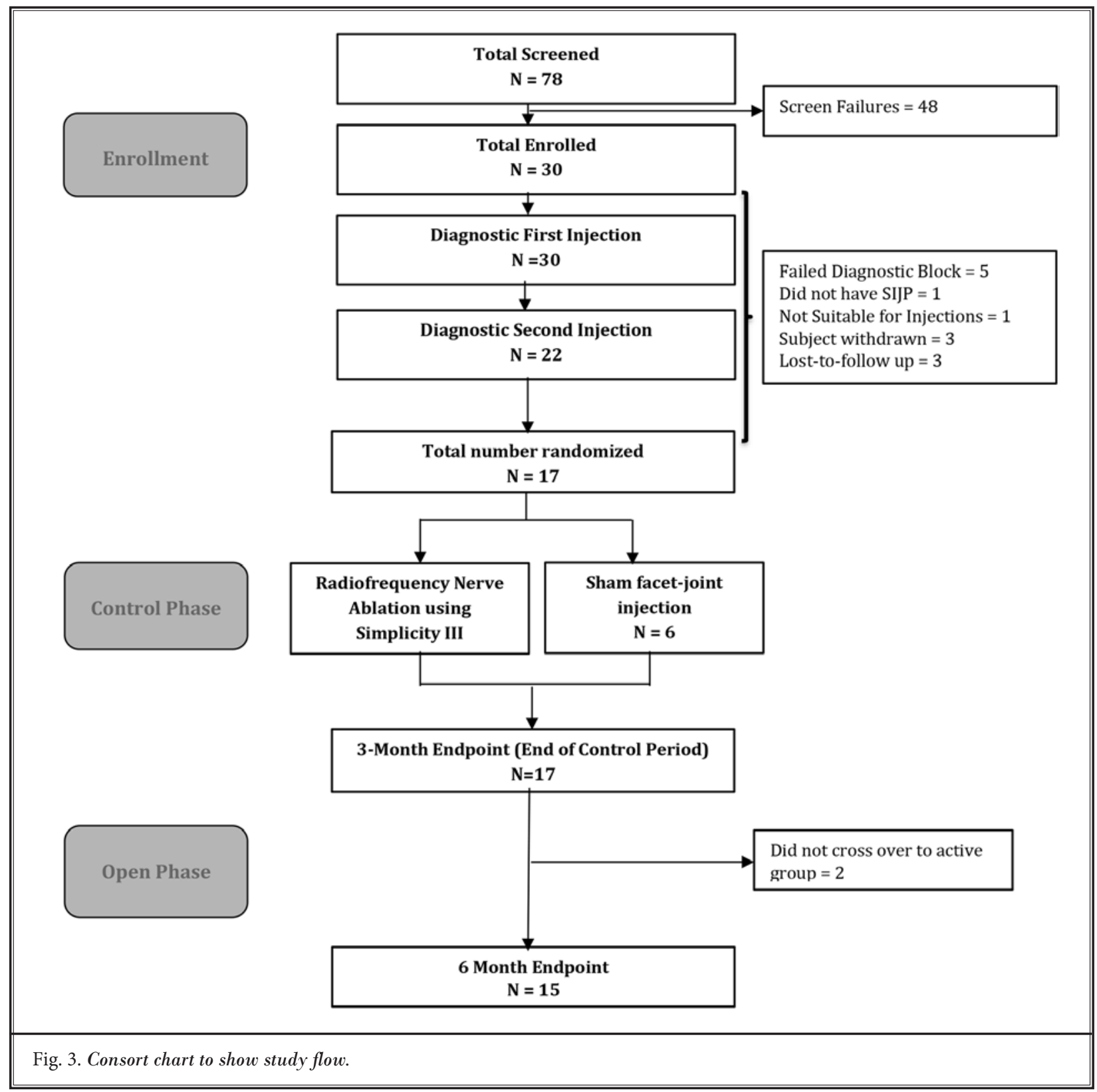

scores in the active group at 3 months, the differences were not statistically different from baseline. Moreover, at 3 months, no statistical differences were found between the active and sham groups for the PCS and MCS $(P=0.0645$ and $P=0.2965$, respectively) (Fig. 4).

\section{Secondary Endpoints: 6-Month Crossover Outcomes}

The mean NRS-11 score reported by all patients (active \pm crossover) was 4.2 , a statistically meaningful reduction from baseline $(P<0.001)$. After crossing over to the active group at 6 months, all patients with RFN treatment reported a mean HAD-Anxiety score of 6.7 \pm 5.0 and mean HAD-Depression score of $8.7 \pm 6.0$. At 6 months, patients reported a mean EQ-5D score of $0.43 \pm 0.33$. Finally, patients reported mean 6-month PCS and MCS scores of $34.7 \pm 10.8$ and $49.1 \pm 9.9$, respectively. Besides the NRS-11, no secondary outcomes demonstrated significant difference from baseline (all $P$ values > 0.05) (Fig. 4). 


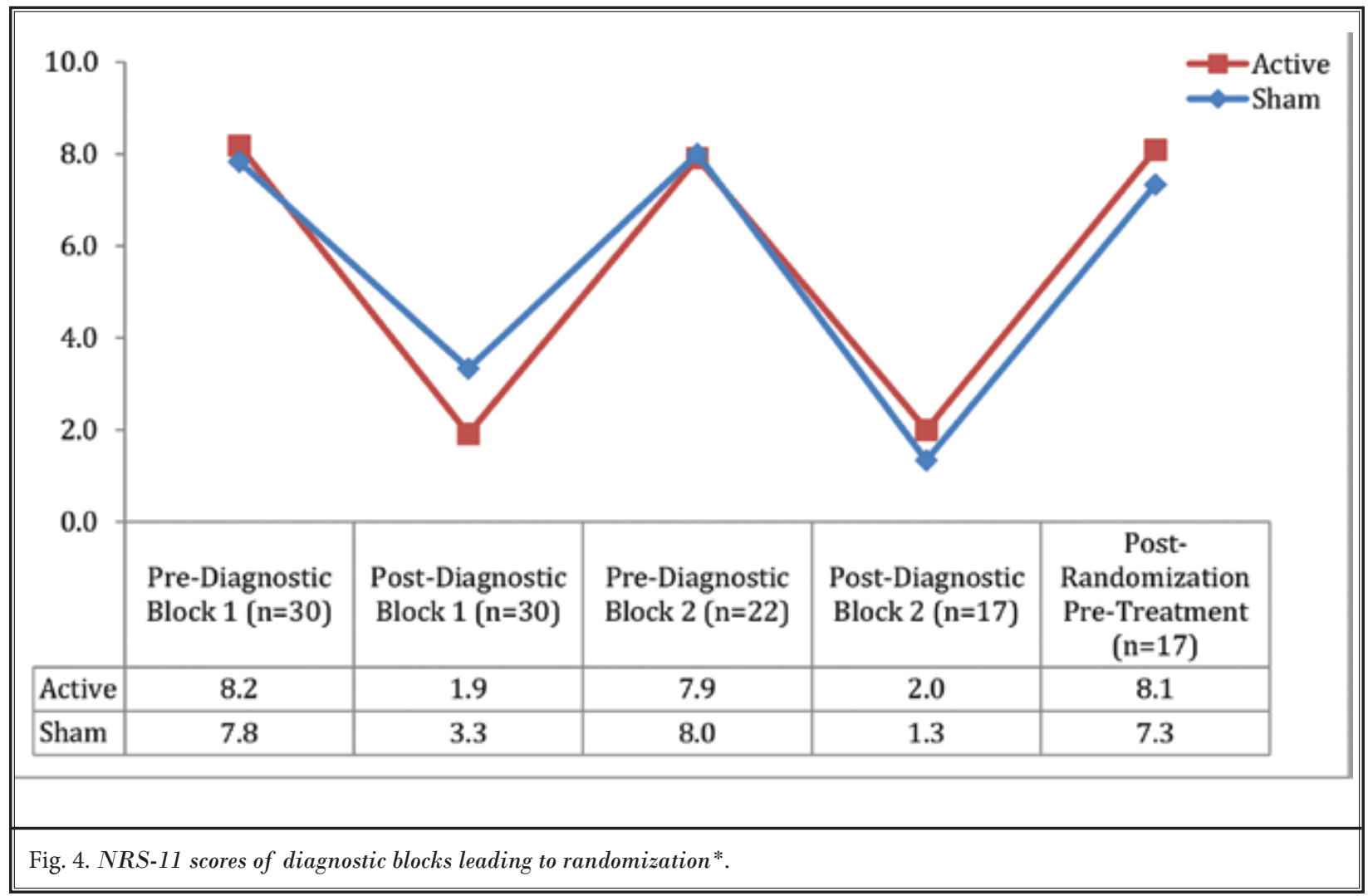

\section{Adverse Events}

There were 8 non-serious AEs reported by 8 patients $(47.0 \%)$ over the course of the study (5 in the active, 3 in the sham group). In the active group, 3 out of the 5 events involved pain at the injection site. One patient developed an L5-S1 disc prolapse on the same side and one patient reported inflammation around the injection site. In the sham group, 1 patient developed pain that extended throughout their back. One patient was diagnosed with asthma during the study and one patient developed rheumatoid arthritis. These events were all addressed and appropriately treated. All 8 patients continued in the study (Table 2).

\section{Discussion}

Besides the prospective nature and strict diagnostic criteria used in this study, its major strength is the use of a true sham procedure, maintaining blinding of patients and investigators. Device and interventional therapy studies are often limited by the nature of the control employed. Placebo effects are seen including the Hawthorne effect (improvement as a result of being evaluated), the Jastrow effect (improvement
Table 2. Adverse events.

\begin{tabular}{|l|c|c||}
\hline \multicolumn{1}{|c|}{ Event } & Active & Sham \\
\hline Pain on site & 3 & 0 \\
\hline Pain extended to all over back & 0 & 1 \\
\hline Asthma diagnosis & 0 & 1 \\
\hline Developed rheumatoid arthritis & 0 & 1 \\
\hline Flare up around site & 1 & 0 \\
\hline $\begin{array}{l}\text { Developed L5-S1 disc prolapse on the same } \\
\text { side }\end{array}$ & 1 & 0 \\
\hline Total events & 5 & 3 \\
\hline
\end{tabular}

secondary to explicit expectation of outcome), and the Halo effect (improvement attributed to the novelty of the intervention). In addition to these well-described biases, blinding with a sham procedure can also be difficult if the treatment has a clear and immediate biological effect, e.g., procedural pain. The RFN from Simplicity III is unique, as the lesion is pain-free with no biofeedback for the patient and, in turn, the operator/ investigator. 

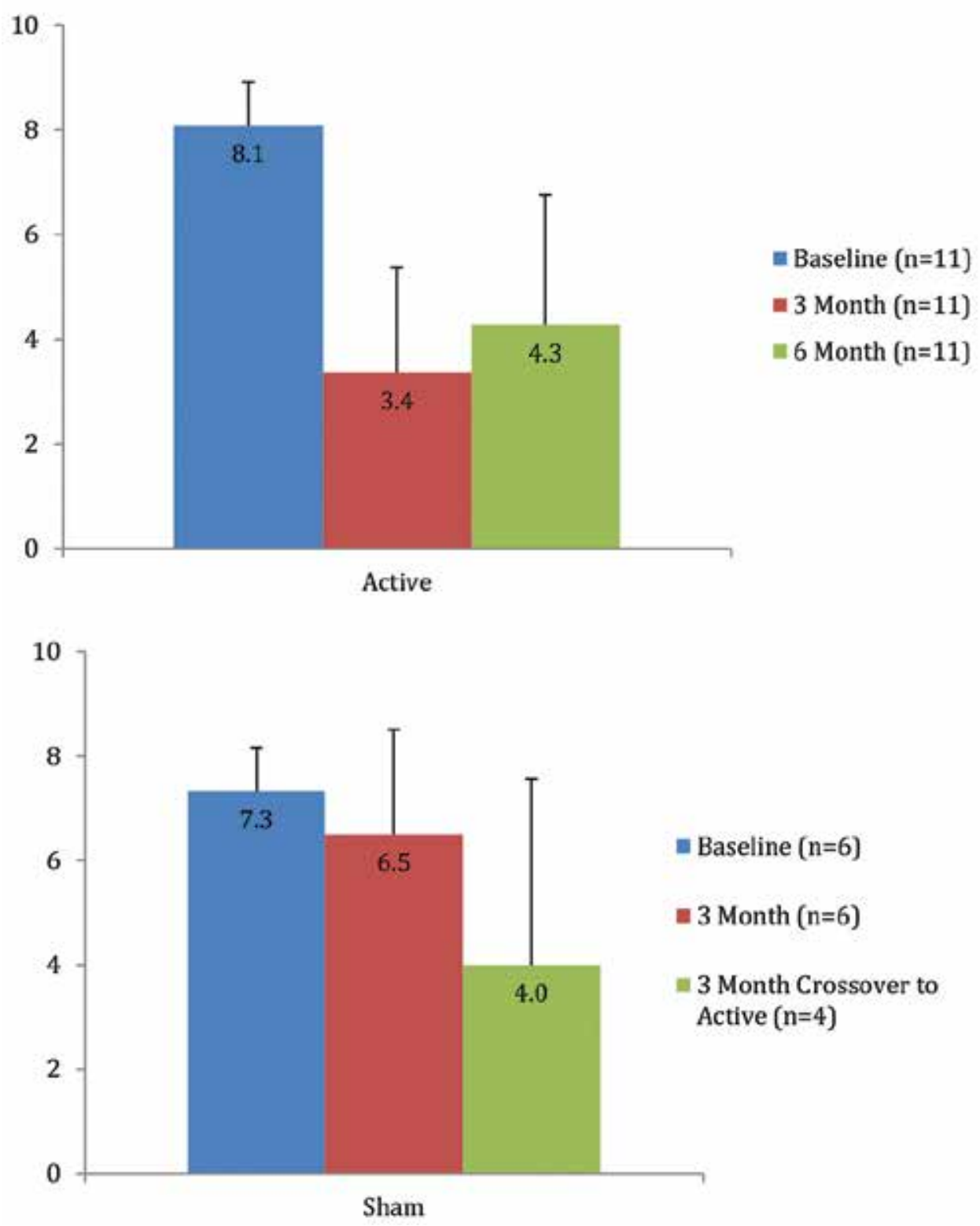

Fig. 5. Primary endpoint-Mean NRS.

National Institute of Clinical Excellence (NICE) guidelines have also lamented the need for shamcontrolled trials in radiofrequency in their recently published guidelines (NG 59, 2016) for low back pain management, although SIJ pain was not in the scope of their review (23). The use of a "true" sham model in this study makes it quite unique.

Our results demonstrate that RFN using the strip lesioning technique significantly improves pain intensity scores in patients for up to 6 months of treatment with nonsignificant improvements in mental health. Patients with RFN therapy achieved a mean NRS-11 decrease of 4.7 at 3 months and sustained this improvement for at least 6 months. Moreover, our study found that patients under sham treatment reported a significant reduction in NRS-11 scores only after crossing over to active treatment. Although no statistically significant improvements were discovered in our secondary out- 


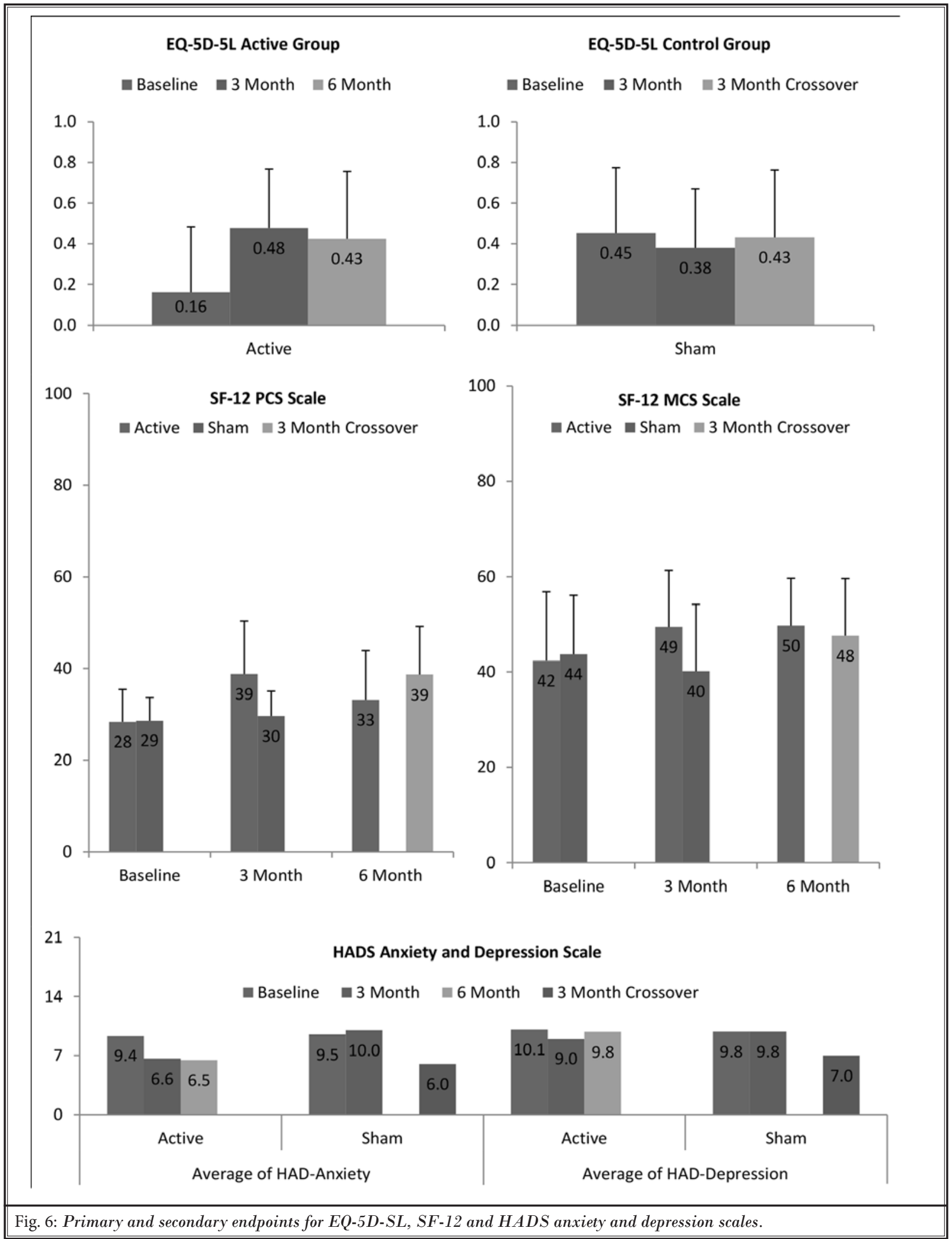


comes, patients in the treatment group, on average, experienced categorical improvements in their anxiety levels from "borderline abnormal anxiety (8-10)" at baseline to "normal levels of anxiety (0-7)" at 3 and 6 months. Combined with no reports of any serious AEs, our results suggest that RFN using the strip lesioning device may provide a therapy with a good risk-benefit profile for patients suffering from SIJP.

Recent advancements in technology have improved neuronal targeting and electrode placement, and have allowed for the production of a larger thermal field using an electrode cooling method. However, these techniques still require multiple injection points and individual neuronal lesioning, extending procedure time and patient burden. RFN using the strip lesioning device is a novel advancement that allows for multiple precise lesions through a single entry point, minimizing both patient and physician burden. Although there have been a few case studies evaluating the benefits of the strip lesioning device, our study is the first randomized, double-blinded, sham-controlled study to provide evidence of 6 months of pain relief from this system.

Our results are similar to the findings of several published studies that evaluated RFN therapy in patients suffering from SIJP $(19,20)$. Using cooled RFN, Patel et al found a statistically significant reduction in patient pain scores, from an average of 6.1 at baseline to an average of 3.5 at 3 months post-intervention. As in our study, there was no significant difference between baseline and 3-month pain scores in the sham group. In a retrospective analysis, Reddy et al found statistical improvements in the SF-12 subscales when using the strip lesioning device. That our study demonstrated trends, but not statistically significant improvements, in the SF-12 subscales may be due to the relatively smaller sample size in our study. The positive trends reported here justify future studies with larger sample sizes, which may replicate significant improvements in these secondary outcomes.

Recently, van Tilburg and colleagues failed to demonstrate any benefit of percutaneous radiofrequency treatment after 3 months in a sham-controlled trial of patients with SIJP. In fact, the proportion of patients who reported significant pain relief (NRS $\geq 2$ ) after the sham procedure was even higher than that of those who received the actual treatment. The researchers attributed this finding to a variety of reasons, such as inclusion criteria resulting in a quite heterogenous patient population that included patients with radicular pain beyond the knee and an unexpectedly high number of patients demonstrating positive responses to SIJ local anesthetic blocks (86.1\%, 62/72 blocks) - much greater than expected from the available literature (21).

The model: We had very stringent criteria for diagnosis of SIJP. Although there are suggestions that the extraarticular part of the SIJ - in particular, the posterior ligaments - may also have some relevance as pain generators in addition to the intraarticular part (24), lack of a precise diagnostic test for SIJP remains a challenge. Currently, the double diagnostic intraarticular joint injection with local anesthetic remains the most commonly practiced tool and may be regarded as the gold standard in diagnosing SIJP syndrome.

We screened our patients using history and clinical examination (unilateral localized lumbosacral pain without any radiculopathy features and a positive provocation pain test), followed by the 2 diagnostic intraarticular local anesthetic injections. Both groups of patients (active and sham) behaved similarly in their diagnostic block responses: a) the mean baseline NRS11 scores were 8.2 and 7.8 , reducing to 1.9 and 3.3 in the active and sham groups at 6 hours at discharge, respectively; and b) the mean NRS-11 scores returned to approximately pre-diagnostic levels, 7.9 and 8.0, respectively, prior to the second injection. For both groups, the second injection improved the pain scores, this time to 2.0 and 1.3 in the active and sham groups, respectively. The fact that NRS-11 scores rose again to 8.1 and 7.3 in the active and sham groups, respectively, following initial positive responses, and prior to randomization, suggests a valid diagnostic method, with equipoise with respect to pain scores before randomization and blinded study treatment (Fig. 4).

Sham group: In this prospective randomized study, true double-blinded sham and assessment methods were implemented, with sham patients receiving the actual probe at the location but not receiving the thermal lesion, and blinding maintained until the 3-month primary objective assessment.

\section{Limitations}

The primary limitations of our study result from the low sample size. The lack of patients in our study limits the strengths of our conclusions. Originally, the study was designed to randomize 30 patients; however, recruitment was stopped after the interim analysis, at 30 enrolled patients, with only 17 randomized, as it was deemed unethical to proceed with sham treatment given the statistically significant $(P<0.001)$ difference in pain outcomes between the treatment and 
sham groups. That active treatment was by then being routinely offered to patients in the UK as part of SIJP treatment provided additional ethical justification for ending recruitment. Further unbalanced allocation with 2:1 randomization was used to hasten recruitment and mitigate patient withdrawal, with the assumption that patients would be more likely to join placebo or sham-controlled trials when their odds of receiving active treatment are greater. Unequal allocation has consequences for statistical power, however, with a 2:1 allocation ratio requiring $12 \%$ more patients to detect the same size effect with equivalent power, compared to a trial using a 1:1 ratio (25).

This study justifies consideration of future multicenter studies evaluating the Simplicity III device in a larger cohort, and with longer term (e.g., 12-month) follow-up. Although several outcomes were collected in this study, other measures that evaluate medica- tion changes (particularly opioid usage) and disability improvements should be included. Lastly, future studies comparing cooled RFN and RFN using Simplicity III should be considered.

\section{Conclusion}

In conclusion, this single-center, prospective, double-blinded, randomized (2:1), sham-controlled trial demonstrated the safety and effectiveness of the Simplicity III device, providing strip lesioning in the treatment of SIJP. At 3 months, the mean NRS-11 score for the active group significantly decreased from 8.1 $( \pm 0.8)$ at baseline to $3.4( \pm 2.0)(P<0.001)$. Patients receiving this therapy reported improved pain scores, less anxiety, and no incidence of a serious AE. This study provides further evidence of the benefits of RFN when conventional treatments prove ineffective.

\section{References}

1. Cohen SP. Sacroiliac joint pain: A comprehensive review of anatomy, diagnosis, and treatment. Anesth Analg 2005; 101:1440-1453.

2. Bernard TN, Cassidy JD. The sacroiliac joint syndrome: Pathology, diagnosis, and management. In: The adul spine principles and practice 2nd edition. Frymoyer, JW (ed). Lippincott-Raven publishers, New York, NY 1991 pg. 2107-2130.

3. Rupert MP, Lee M, Manchikanti L, Datta S, Cohen SP. Evaluation of sacroiliac joint interventions: A systematic appraisal of the literature. Pain Physician 2009; 12:399-418.

4. Hansen, HC, McKenzie-Brown AM, Cohen, SP, Swicegood JR, Colson JD, Manchikanti L. Sacroiliac joint interventions: A systematic review. Pain Physician 2007; 10:165-184.

5. Zelle BA, Gruen GS, Brown S, George S. Sacroiliac joint dysfunction: Evaluation and management. Clin J Pain 2005; 21:446-455.

6. Fischer T, Biedermann T, Hermann KG, Diekmann F, Braun J, Hamm B, Bollow M. Sacroiliitis in children with spondyloarthropathy: Therapeutic effect of CT-guided intraarticular corticosteroid injection [in German]. Rofo 2003; 175:814-821.
7. Van Tulder MW, Koes BW, Bouter LM. Conservative treatment of acute and chronic nonspecific low back pain: A systematic review of randomized controlled trials of the most common interventions. Spine 1997; 22:2128-56

8. Shealy NC. Percutaneous radiofrequency denervation of spinal facets: Treatment for chronic back pain and sciatica. ] Neurosurg 1975; 43:448-451.

9. Leggett LE, Soril LJ, Lorenzetti DL, Noseworthy T, Steadman R, Tiwana S, Clement $F$. Radiofrequency ablation for chronic low back pain: A systematic review of randomized controlled trials. Pain Res Manag 2014; 19:e146-e153.

10. Maas ET, Ostelo RWJG, Niemisto L, Jousimaa J, Hurri H, Malmivaara A, van Tulder MW. Radiofrequency denervation for chronic low back pain. Cochrane Database Syst Rev. 2015; (10):CDoo8572.

11. Lakemeier S, Lind M, Schultz W, FuchsWinkelmann S, Timmesfeld N, Foelsch C, Peterlein CD. A comparison of intraarticular lumbar facet joint steroid injections and lumbar facet joint radiofrequency denervation in the treatment of low back pain: A randomized, controlled, double-blind trial. Anesth Analg 2013; 117:228-235.

12. Yin W, Willard F, Carreiro J, Dreyfuss P. Sensory stimulation-guided sacro- iliac joint radiofrequency neurotomy: Technique based on neuroanatomy of the dorsal sacral plexus. Spine 2003; 28:2419-2425.

13. Fortin JD, Kissling RO, O'Connor BL, Vilensky JA. Sacroiliac joint innervation and pain. Am J Orthop 1999; 28:687-690.

14. Grob KR, Neuhuber WL, Kissling RO. Innervation of the sacroiliac joint of the human. Z Rheumatol 1995; 54:117-122.

15. Kapural L, Vrooman B, Sarwar S, Krizanac-Bengez L, Rauck R, Gilmore C, North J, Girgis G, Mekhail N. A randomized, placebo-controlled trial of transdiscal radiofrequency, biacuplasty for treatment of discogenic lower back pain. Pain Med 2013; 14:362-373.

16. Kvarstein G, Mawe L, Indahl A, Hol PK, Tennoe B, Digernes R, Stubhaug A, Tonnessen TI, Beivik $\mathrm{H}$. A randomized double-blind controlled trial of intraannular radiofrequency thermal disc therapy - a 12-month follow-up. Pain 2009; 145:279-286.

17. Leclaire $\mathrm{R}$, Fortin L, Lambert $\mathrm{R}$, Bergeron YM, Rossignol M. Radiofrequency facet joint denervation in the treatment of low back pain: A placebocontrolled clinical trial to assess efficacy. Spine 2001; 26:1411-1416.

18. Cohen SP, Hurley RW, Buckenmaier CC 
III, Kurihara Connie, Morlando Benny, Dragovich Anthony. Randomized placebo-controlled study evaluating lateral branch radiofrequency denervation for sacroiliac joint pain. Anesthesiology 2008; 109:279-288.

19. Patel N, Gross A, Brown L, Gekht G. A randomized, placebo-controlled study to assess the efficacy of lateral branch neurotomy for chronic sacroiliac joint pain. Pain Med 2012; 13:383-398.

20. Reddy VSA, Sharma C, Chang K, Mehta $V$. 'Simplicity' radiofrequency neurotomy of sacroiliac joint: A real life 1-year follow-up UK data. Br J Pain 2016; 10:90-99.
21. van Tilburg CW, Schuurmans FA, Stronks DL, Groeneweg JG, Huygen FJ. Randomized sham-controlled doubleblind multicenter clinical trial to ascertain the effect of percutaneous radiofrequency treatment for sacroiliac joint pain: Three-month results. Clin J Pain 2016; 32:921-926.

22. Bogduk N (ed). Practice Guidelines for Spinal Diagnostic and Treatment Procedures. 2nd ed. Spine Intervention Society, San Francisco, CA, 2013.

23. National Institute of Clinical Excellence. Low back pain and sciatica in over $16 \mathrm{~s}$ : Assessment and management. www. nice.org.uk/guidance/ng59. Published
November 30, 2016. Accessed September 18, 2017.

24. Hartung W, Ross CJ, Straub R, Feuerbach S, Scholmerich J, Fleck M, Herold T. Ultrasound-guided sacroiliac joint injection in patients with established sacroiliitis: Precise IA injection verified by MRI scanning does not predict clinical outcome. Rheumatology 2010; 49:1479-1482.

25. Avins AL. Can unequal be more fair? Ethics, subject allocation, and randomised clinical trials. J Med Ethics 1998; 24:401-408. 